\title{
A Self-Aligning Cylindrical Sliding Short Plunger for Millimeter-Wave Rectangular Waveguides and its Application in a Reflection-Type Phase Shifter
}

\author{
Christian Koenen, Student Member, IEEE, Uwe Siart, Member, IEEE, Thomas F. Eibert, Senior Member, IEEE, \\ and Garrard D. Conway
}

\begin{abstract}
A sliding short for millimeter-wave rectangular waveguides and its dedicated application in a reflection-type phase shifter are presented. The design of the sliding short has self-aligning properties, where the correct and stable positioning of the sliding short on the central axis of a rectangular waveguide is guaranteed. Unwanted and disruptive waveguide wall contacts are avoided by design. Misalignment of the sliding short is only possible due to mechanical tolerances. This is systematically investigated by parametric full-wave simulations. Measurements of a $W$-band prototype yield a return loss better than $0.1 \mathrm{~dB}$ for offset positions from $0 \mathrm{~mm}$ to $1.5 \mathrm{~mm}$ throughout the whole $W$ band. A mechanical reference plane outside of the rectangular waveguide is used for correct and reproducible axial positioning of the sliding short in the waveguide. Mechanically measured offset positions and those retrieved from the reflected phase agree to a maximum error of $6 \mu \mathrm{m}$ which is within the manufacturing tolerance and the measurement uncertainty. Based on the proposed self-aligning sliding short, a reflection-type phase shifter has been realized, where a branch-guide coupler with a 7-slot design is used as broadband quadrature hybrid. Measurement results indicate an insertion loss in the range of $0.2 \mathrm{~dB}$ from $80 \mathrm{GHz}$ to $105 \mathrm{GHz}$ and a maximum phase shift of approx. $160^{\circ}$ and $330^{\circ}$ at $75 \mathrm{GHz}$ and $110 \mathrm{GHz}$, respectively.
\end{abstract}

Index Terms-Micromachining, millimeter-wave passive components, phase shifters, millimeter-wave circuits, millimeter-wave devices, waveguide components

\section{INTRODUCTION}

$\mathbf{S}$ TEERABLE phase shifting circuits are needed in a variety of applications such as adaptive tuning units or beamsteered phased array antennas. There are numerous mechanical and electronic technologies to realize a passive variable phase shifter. These can generally be categorized by three prevailing topologies: Switched-line, loaded-line, and reflection-type phase shifters. In switched-line phase shifters, the electrical length from the input to the output port is changed by internally switching between waveguides of different length. PIN diodes, field-effect transistors or microelectromechanical

Manuscript received Month dd, yyyy; revised Month dd, yyyy.

The authors express their gratitude to the Helmholtz Association of German Research Centres for funding this work within the framework of the Helmholtz Virtual Institute on "Plasma Dynamical Processes and Turbulence Studies using Advanced Microwave Diagnostics". They are also indebted to the foreman of the precision mechanical workshop Josef Franzisi for helpful suggestions and Clemens Moroder for initial investigations towards a millimeter-wave reflection-type phase shifter.

C. Koenen, U. Siart, and T. F. Eibert are with the Chair of High-Frequency Engineering of the Technical University of Munich, 80290 München, Germany

G. D. Conway is with the Max Planck Institute for Plasma Physics, 85748 Garching, Germany systems (MEMS) are used for this purpose [1]-[3]. Loadedline phase shifters aim at changing the propagation constant of a certain waveguide section. This is done e.g., by periodic loading of the transmission line with shunt varactor diodes or MEMS, by variably modifying the permeability with a ferrite or the permittivity with liquid crystals [4]-[6]. Reflection-type phase shifters use circulators or hybrid junctions to transform a variable and reflective load into transmission from the input to the output port. Suitable reflective loads are switched transmission lines, varactor diodes or sliding shorts [6]-[9].

The approach proposed in this manuscript has been motivated by the design and development of a millimeter-wave phased array antenna as communicated in [10]-[12]. Such kind of antennas are required for observation and study of turbulent plasma phenomena in nuclear fusion experiments [13], [14]. The underlying measurement principle is Doppler reflectometry. By use of a steered Gaussian beam it becomes possible to measure wavenumber $k$-spectra of backscattered micro or mm-waves. These yield information about the spatial structure of the turbulent flows [15]-[17]. However, the usability of the proposed approach is certainly not limited to this particular application. In view of the essential requirements for in-vessel use within nuclear fusion experiments, any component has to comply with and sustain the corresponding environmental conditions. These are ultra-high vacuum $\left(10^{-9} \mathrm{mbar}\right)$, temperatures up to $160^{\circ} \mathrm{C}$, strong magnetic fields $(3.9 \mathrm{~T}$ on ASDEX Upgrade tokamak), and the presence of neutron radiation. The phase shifter should operate from $75 \mathrm{GHz}$ to $105 \mathrm{GHz}$ and it should have a low insertion loss. The maximum required phase shift is around $200^{\circ}$ at $105 \mathrm{GHz}$ and continuously varying the phase state should be possible.

Switched-line and loaded-line phase shifters do obviously not comply with these requirements, but reflection-type phase shifters appear to be very suitable. They can be built predominantly from metal. The hybrid junction can be realized in milled split-block technology as a branch-guide coupler covering the required frequency range (e.g. [18], [19]). The variable reflective load should be a sliding short, because of the harsh environment and the required continuous phase shift. For a serially fed phased array antenna, every phase shifter in a branch has to provide the same phase delay in order to achieve a progressive phase at the array aperture. Consequently, every sliding short has to share the same reference plane. The design of the sliding short should allow for a manufacturing process that can fulfill highest demands on mechanical tolerances and 
thus provide low variation between the separate plungers.

In view of the described requirements, a reflection-type phase shifter is proposed, which is based on a branch-guide coupler as hybrid junction and an all-metal circular cylindric plunger that can be manufactured on a lathe. Manufacturing on a lathe provides sharp edges and good contour accuracy (in the range of $\pm 5 \mu \mathrm{m}$ in our mechanical workshop). The plunger is mechanically aligned, supports a dedicated reference plane and it is guided inside a fitting hole. This keeps the plunger centered and ensures linear motion. It also prevents unwanted pitching and tangency with the waveguide walls even during mechanical movement. Strictly speaking, this concept is both contacting and noncontacting. The electrically effective part of the plunger is noncontacting whereas the part where the fields have already decayed is contacting, for the sake of mechanical stability. The contact is for mechanical purposes only and its electrical characteristics are completely irrelevant for the electrical performance of the sliding short. A Piezo actuator can be employed to position the reference plane at the desired position.

The paper is structured as follows. In section II, the design of the sliding short is presented and assorted into the state-ofthe-art. The impact of residual misalignment of the plunger is investigated in section III by means of parametric full-wave simulations. Measurement results from a $W$-band prototype sliding short are presented in section IV. The design and measurements of the reflection-type phase shifter are given in section $\mathrm{V}$ and section VI, respectively.

\section{SLIDING ShORT DESIGN}

There are two basic concepts to realize a sliding short. The plunger may be either contacting or noncontacting. Contacting sliding shorts are not very common at millimeter-wave frequencies because there are a few drawbacks. Their electrical properties would depend significantly on the quality and the position of the physical ground short. If the ground short is realized by a sliding contact it may vary in an unpredictable manner due to surface roughness and frictional wear [20], [21]. This yields random variations in reflection coefficient amplitude and phase. Furthermore, parasitic power leakage and radiation may occur [21]. Noncontacting sliding short circuits for millimeter-wave rectangular waveguides have been built either from metal only [20]-[23] or by use of dielectric materials as insulator between plunger and waveguide [20], [24], [25]. A dielectric slab to change the phase velocity of the guided wave over a variable length is a further possible approach [26]. However, sliding shorts for in-vessel use in fusion experiments must avoid any dielectric materials due to unacceptable gas emission. Sliding shorts at millimeterwave frequencies are very often based on a quasi-periodic choke structure which realizes a transmission stop-band using alternating low-impedance and high-impedance sections [20], [21], [23]-[25]. The choke structure may have either a rectangular or a circular cross section [21]. In either case, the central axis of the choke structure should coincide with the central axis of the rectangular waveguide for best performance and to avoid unwanted contact to the waveguide wall. This is either achieved by an external mounting frame, by insulating dielectric, or by directing the plunger in a fitting groove [21], [23]-[25]. In particular, the very interesting design in [23] can, however, not be manufactured on a lathe, due to its rectangular shape, and it does not provide a well defined reference plane, which is very important for the synchronized control of several short-circuit plungers.

The basic concept of the proposed design is to use a cylindrical plunger inside the rectangular waveguide. This essentially enables mechanical guidance and stabilization by a fitting hole (see Fig. 1). The plunger is made up of three principal elements. There is a noncontacting choke section, a contacting section, and a mechanical stop that serves as a reference plane for positioning. The noncontacting section is a coaxial periodic structure that consists of alternating low-impedance and high-impedance sections. It effectuates an electrical short in the transverse plane at the very left end of the plunger (see Fig. 2). The contacting section provides a means for directing the plunger in the fitting hole of the fundamental waveguide (see Fig. 1 and Fig. 2). Thereby, random and unwanted contact of the noncontacting choke structure is prevented. For controlling the position of the sliding short, a reference plane, which is located outside the hollow waveguide, is used. For phased array applications, all sliding shorts can be mounted on a single mounting frame and, thus, share the same reference plane. Thereby, it can be guaranteed that all sliding shorts are located at the same axial position and, thus, entail identical phase shifts.

Because the plunger is a turning workpiece, it can be manufactured on a lathe. This offers three advantages. The manufactured element is circularly symmetric and, thus, the fitting hole will always position the choke structure correctly centered in the waveguide - provided that the fitting hole itself is correctly centered within the waveguide. Furthermore, the manufacturing process of the sliding short starts on the left side (compare Fig. 2). The machine can have its reference position there. From this reference position to the reference plane of the sliding short, the lathe tool just needs to move a certain delta in the direction of the rotation axis. Thereby, the accuracy of the reference plane and also that of the contour of the sliding

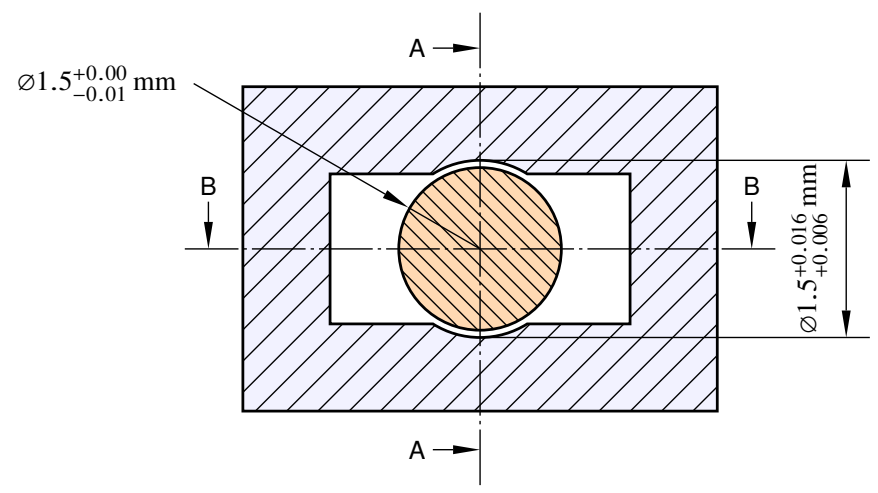

Figure 1. Cross sectional view of the sliding short. The plunger is directed and stabilized in the center of a WR 10 waveguide $(1.27 \mathrm{~mm} \times 2.54 \mathrm{~mm})$ by a fitting hole. The gap between plunger shaft and fitting hole is supposed to prevent sticking and clamping during temperature variations. 


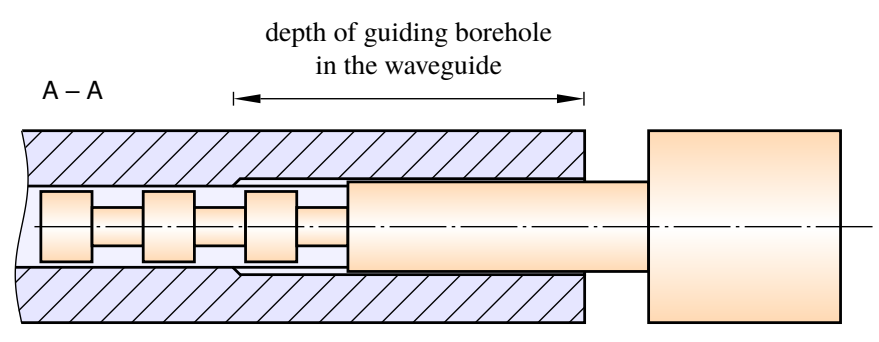

$B-B$

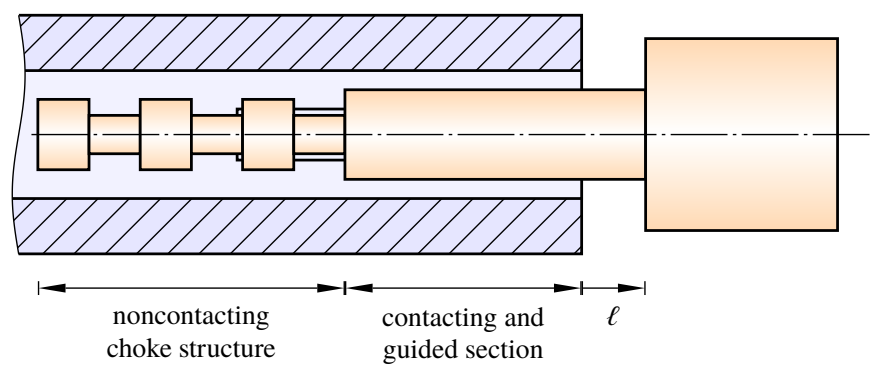

Figure 2. Longitudinal cut of the sliding short. The noncontacting steppedimpedance choke section implements a short referenced at the very left end of the plunger. For mechanical guidance, a contacting section is integrated behind the choke section. A mechanical stopper defines the reference plane for positioning.

short is equivalent to the machine accuracy $( \pm 5 \mu \mathrm{m}$ in our workshop). Lastly, the transitions from low-impedance to highimpedance section and vice versa can be manufactured with sharp edges in contrast to rounded edges that would appear in manufacturing by laser-cutting or etching.

The design of the noncontacting choke section is based on the traditional $\lambda / 4$ design and not on, e.g., the low-pass filter technique employed in [20]. The reason for this is that operation in a single waveguide band is aimed and the $\lambda / 4$ design seems to be superior to the low-pass filter technique in this smaller frequency band [20]. The lengths of the individual impedance sections of the sliding short are $0.8 \mathrm{~mm}$ which corresponds to approximately $\lambda / 4$ at the design frequency $92.5 \mathrm{GHz}$. The diameter of the low-impedance section is $1.15 \mathrm{~mm}$ and that of the high-impedance section is $0.6 \mathrm{~mm}$. This choice is mainly driven by mechanical producibility. In electrical terms, the sliding short performance improves for larger impedance discontinuities [20]. The diameter of the lowimpedance section is as large as possible, but still such that it will never contact with the waveguide walls due to mechanical tolerances and misalignment. The high-impedance section is chosen as thin as possible so that the electrical performance achievable is satisfactory while mechanical stability is still guaranteed. The decision on the high-impedance diameter was underpinned by a full-wave simulation analysis. The fitting hole is reamed on the center line of the rectangular hollow waveguide with a diameter of $1.5 \mathrm{~mm}$ and ISO 286 tolerance grade on hole F7. The guided section of the plunger has a length of $4.5 \mathrm{~mm}$ and a diameter of $1.5 \mathrm{~mm}$ with ISO 286 tolerance grade on shaft $\mathrm{h} 7$ so as to provide a clearance fit, see Fig. 1. The entire geometry of the sliding short and of the waveguide is chosen such that no wall contact of the choke structure can occur, see Fig. 2. There will only be wall contact
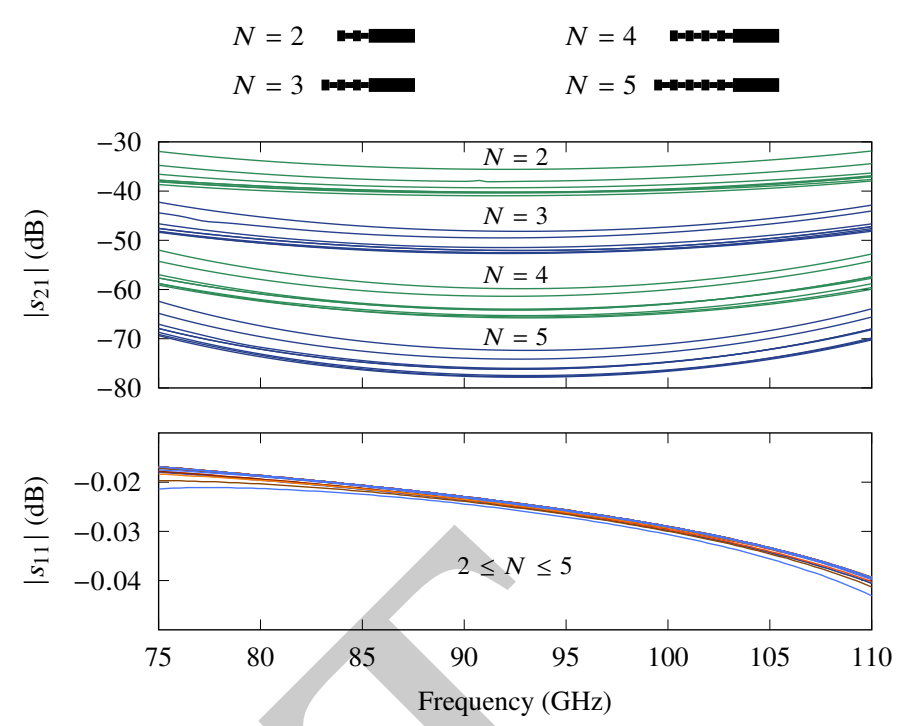

Figure 3. Impact of length $N$ of the choke structure and steering position $\ell$ on reflectivity $\left(\left|s_{11}\right|\right)$ and back end transmission $\left(\left|s_{21}\right|\right)$. The steering position $\ell$ increases from $0.00 \mathrm{~mm}$ to $1.50 \mathrm{~mm}$ from the lowest transmission to the largest for each $N$. The reference plane for simulation is placed $0.1 \mathrm{~mm}$ in front of the plunger for all steering positions and choke structure variations. Thereby introduced additional Ohmic losses are neglected. Waveguide filling is vacuum, waveguide walls have a finite conductivity of $15.9 \times 10^{6} \mathrm{~S} / \mathrm{m}$ (brass) and the plunger has a conductivity of $5 \times 10^{6} \mathrm{~S} / \mathrm{m}$ (nickel silver).

within the guiding section, where the field has already decayed through the choke structure. Therefore, this contact is of no significance for the electrical performance of the sliding short.

A simulation study to support this statement is carried out in section III. Since a sliding short plunger is supposed to totally reflect the incident power, the amount of power that appears at the back end of the plunger (right hand side in Fig. 2) may also be used as a predictor of functionality. Here, it is measured by the $\left|s_{21}\right|$ parameter of the dominant coaxial $\mathrm{TE}_{11^{-}}$ like mode [21]. Back end leakage is influenced predominantly by two factors. First, there are the diameters of the lowimpedance and high-impedance sections. Secondly, the length of the choke segment, i.e. the number $N$ of subsequent lowimpedance high-impedance sections. In Fig. 3, the dependency of back end leakage $\left|s_{21}\right|$ on the number of impedance sections $N$ and the choke position $\ell$ is depicted. It should be noted that $\left|s_{21}\right|$ has been determined by waveguide port analysis and not by numerical computation of the radiated power. Assuming that there is always open end reflection, the value of $\left|s_{21}\right|$ may be considered as a worst case measure for parasitic back end leakage radiation. The reference plane for the $s_{11}$ is positioned $0.1 \mathrm{~mm}$ apart from the first choke section for all plunger configurations, and, thus, suppressing the influence of slightly higher Ohmic waveguide losses for larger $\ell$. The diameter of the low-impedance section is $1.15 \mathrm{~mm}$ and that of the high-impedance section is $0.6 \mathrm{~mm}$ for this investigation and the plunger is centrally aligned in the rectangular waveguide (i.e. no misalignment). While there is a significant dependency between $N$ and the overall level of back end leakage, the leakage is almost independent of the choke position. A longer choke segment further reduces back end leakage, but it also entails additional manufacturing effort and higher cost of 

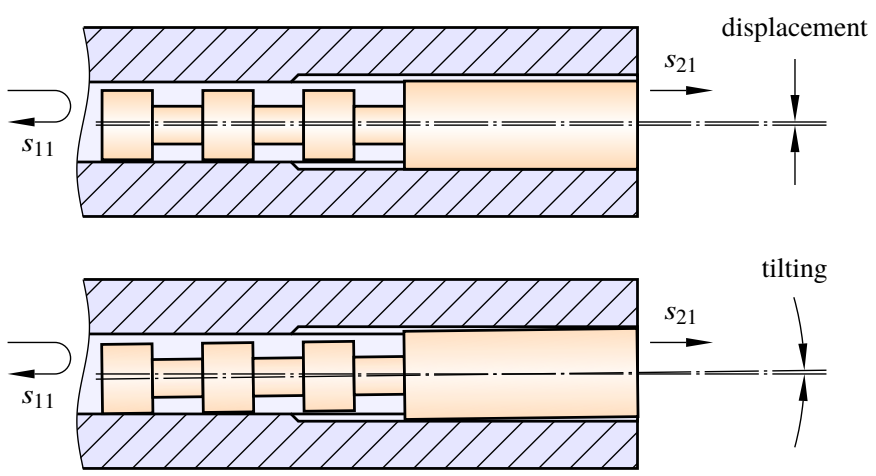

Figure 4. Simulation model of the sliding short in misplaced positions. The reference plane for simulation is placed $0.1 \mathrm{~mm}$ in front of the plunger for all steering positions and choke structure variations. Thereby introduced additional Ohmic losses are neglected. The waveguide filling is vacuum, the waveguide walls have a finite conductivity of $15.9 \times 10^{6} \mathrm{~S} / \mathrm{m}$ (brass), and the plunger has a conductivity of $5 \times 10^{6} \mathrm{~S} / \mathrm{m}$ (nickel silver).

production. The effect of $N$ and $\ell$ on $\left|s_{11}\right|$ is also depicted in Fig. 3. There is no dependency on $N$ and $\ell$, or rather it is too weak to be seen in this coefficient. To reduce back end leakage to an acceptable level while keeping the structure as simple as possible in terms of manufacturing, the number of choke structures is chosen to be $N=3$. This means 3 low-impedance and 3 high-impedance sections. This yields a back end leakage of $40 \mathrm{~dB}$ below the input power over the whole $W$-band, see Fig. 3. Fewer sections implicate higher leakage and vice versa. As mentioned before, the diameters of the individual choke sections also exert influence on the choke behavior. In particular, leakage gets smaller for larger diameters of the low-impedance section as well as for smaller diameters of the high-impedance section. However, the $1.15 \mathrm{~mm}$ diameter of the low-impedance section can not be chosen much larger in this design as the waveguide walls are separated by $1.27 \mathrm{~mm}$. The $0.6 \mathrm{~mm}$ diameter of the high-impedance section is a compromise between mechanical stability of the choke structure and its high-frequency behavior.

\section{Numerical Study of Sliding Short MisALIGNMENT}

In this section, the impact of deviations of the sliding short from its ideal centered position is analyzed. Such kind of error may occur due to tolerances in design and manufacturing. To ensure reliable gliding at all working temperatures and in ultra-high vacuum, the fit along the gliding section cannot be perfectly tight. As a result, the plunger is in a way loosely guided and its axis may become inclined relative to the waveguide axis. In this case, the center of rotation lies in the middle of the contacting section. When the plunger is dragged outward, the center of the contacting section moves in the same direction, but by only half of the plunger displacement (compare Figs. 2 and 4). The potential deflection is largest when the immersion length of the guided section is shortest. This is the case, when the plunger has moved to its outer dead-stop position ( $\ell=1.50 \mathrm{~mm}$ for this design).

Actually, there are two possible kinds of misalignment. The central axes of plunger and waveguide may have some translational offset relative to each other. The second possible error is tilting of the plunger relative to the waveguide central axis. Figure 4 illustrates these two kinds of misalignment. In addition to this, the offset or tilted plunger gets to rotate around the waveguide central axis in its misaligned position. To explore the electromagnetic effects of these two misalignment scenarios empirically, a parameter study based on fullwave simulations using version 2015.6 of CST Microwave Studio ${ }^{\circledR}$ has been carried out. The simulation results of the offset and rotation scenario are plotted in Figs. 5 and 6, respectively. After the last adaptive meshing pass, the change in S-parameter (maximal deviation of the absolute value of the complex difference) was smaller than 0.01 for every parametric simulation. Due to displacement or rotation, symmetry and homogeneity are destroyed and higher order modes can exist [21]. The depicted $\left|s_{21}\right|$ represents the total field of the first five modes at the transition from the plunger to freespace and it is a measure for how good the short chokes and reflects an incident $\mathrm{TE}_{10}$ wave. Both subplots in Figs. 5 and 6 show the maximum and minimum values which have occurred in the frequency range from $75 \mathrm{GHz}$ to $110 \mathrm{GHz}$ of all rotated positions for different axial plunger settings. The behavior over frequency is similar to that shown in Fig. 3 for all misalignment scenarios.

For modeling the offset scenario, the sliding short was placed at maximum offset from the waveguide center, such that it intersects with the guiding fitting hole by $5 \mu \mathrm{m}$. This marginal artifice is necessary because meshing cannot cope with point-like or linear contact. In this position, the sliding short is rotated around the central axis of the rectangular hollow waveguide in angular increments of $1^{\circ}$ in order to emulate all possible misalignment scenarios. With such kind of parameter sweep, full-wave simulations were run for 7 different axial positions of the plunger, i.e. $0 \mathrm{~mm}, 0.25 \mathrm{~mm}, 0.5 \mathrm{~mm}$, $0.75 \mathrm{~mm}, 1 \mathrm{~mm}, 1.25 \mathrm{~mm}$ and $1.5 \mathrm{~mm}$. The simulation results are depicted in Fig. 5. The maximum value of $\left|s_{21}\right|$ that occurs due to displacement of the sliding short from the waveguide center remains lower than $-35 \mathrm{~dB}$ for all plunger positions. Slightly more power is transmitted through the short to the back end for the outermost axial positions. The log reflectivity $20 \lg \left\{\left|s_{11}\right|\right\}$ reduces for larger values of $\ell$. This is an expected result that can be explained by increasing transmission line losses as the waveguide section between waveguide port and plunger is lengthened by $\ell$ (waveguide walls were modeled with a finite conductivity of $15.9 \times 10^{6} \mathrm{~S} / \mathrm{m}$ ). There is no significant variation of the phase of $s_{11}$ over the whole $W$ band range and over all rotation steps (worst case $\pm 0.04^{\circ}$ at $75 \mathrm{GHz}$ and $\pm 0.1^{\circ}$ at $110 \mathrm{GHz}$ ).

The second scenario investigates maximal tilting of the centerlines relative to each other. Again, the plunger is intersecting with the waveguide walls by $5 \mu \mathrm{m}$ and the simulations have been carried out with $1^{\circ}$ rotation steps around the central axis of the rectangular hollow waveguide for the 7 different axial positions in the waveguide. Maximum and minimum values of $\left|s_{21}\right|$ and $\left|s_{11}\right|$ over the frequency range $75 \mathrm{GHz}$ to $110 \mathrm{GHz}$ and all rotation steps are plotted in Fig. 6. The transmission of the incident wave amplitude is choked down by at least $28 \mathrm{~dB}$. Similar to the displaced alignment, the amplitudes of 

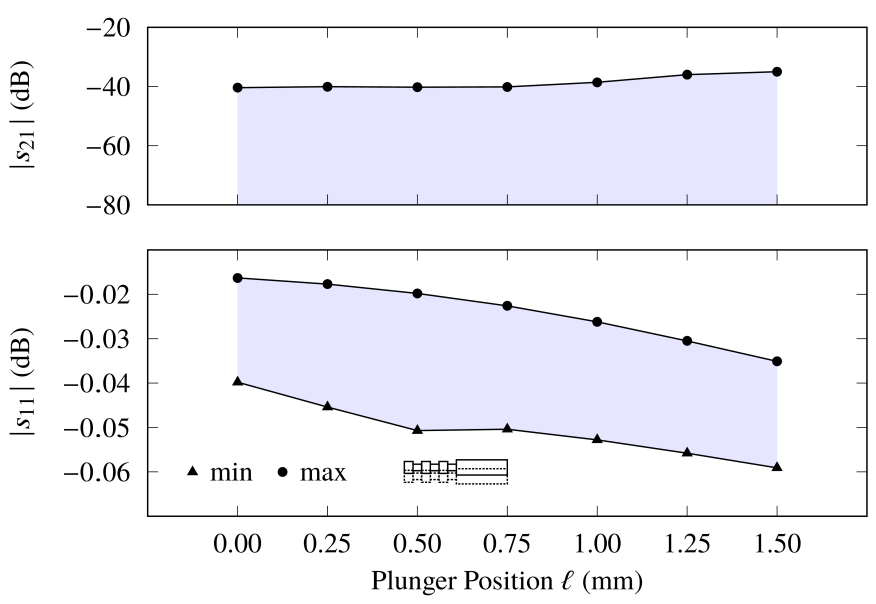

Figure 5. Simulated range of variations of $\left|s_{11}\right|$ and $\left|s_{21}\right|$ due to displacement of the plunger axis. Depicted values represent the minimum and maximum values that occurred over all rotation steps around the waveguide central axis in the misaligned position and in the frequency range from $75 \mathrm{GHz}$ to $110 \mathrm{GHz}$.
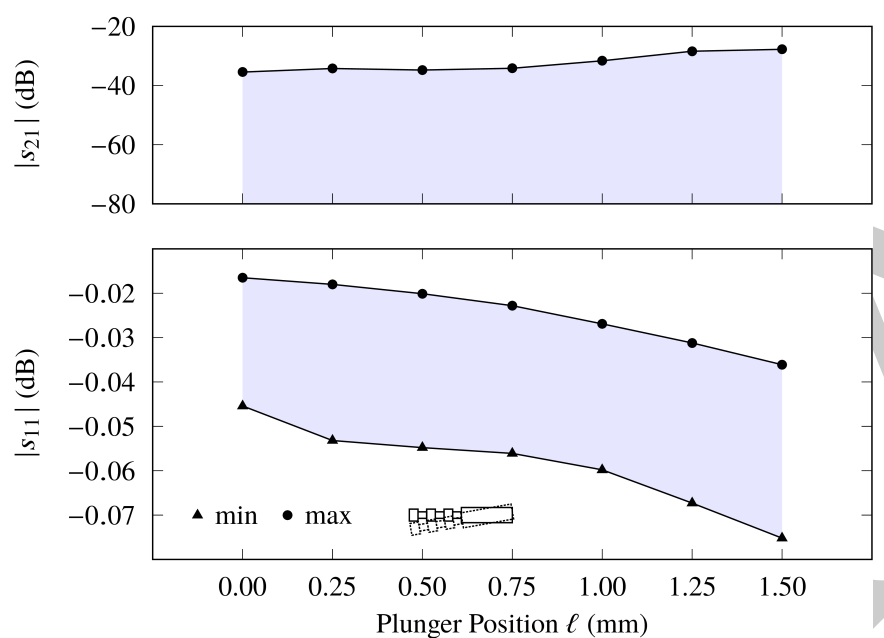

Figure 6. Simulated range of variations of $\left|s_{11}\right|$ and $\left|s_{21}\right|$ due to tilting of the plunger axis. Depicted values represent the minimum and maximum values that occurred over all rotation steps around the waveguide central axis in the misaligned position and in the frequency range from $75 \mathrm{GHz}$ to $110 \mathrm{GHz}$.

$s_{11}$ reduce for larger plunger positions and the reason again is the longer path length from waveguide port to plunger and accompanied higher Ohmic losses. The variation of the phase of $s_{11}$ over the whole $W$-band range and over all rotation steps is slightly stronger than for the displacement scenario, but still relatively small (worst case $\pm 0.6^{\circ}$ at $75 \mathrm{GHz}$ and $\pm 1.3^{\circ}$ at $110 \mathrm{GHz})$.

In summary, variability of the electrical properties of the sliding short by reason of mechanical impreciseness can be expected to remain within the presented margins. The investigated misalignment scenarios have a small impact on the sliding short performance but these are mainly due to higher Ohmic losses as the path length is elongated. Displacement of the plunger and waveguide axis relative to each other as well as tilting of the plunger do not raise the transmission amplitude to unacceptably high values. The influence on the reflected phase is negligible.

\section{Sliding Short Prototype}

A $W$-band prototype of the proposed sliding short is depicted in Fig. 7 and Fig. 8. The rectangular waveguide is manufactured in split-block technology, with the splitting plane lying in the middle of the broad wall edge. Before milling and assembly, the guiding fitting hole was drilled into the split-block. The split-block is made from brass with a milling accuracy on the order of $\pm 10 \mu \mathrm{m}$. The material of the plunger is nickel silver and it was manufactured on a lathe with similar accuracy. Nickel silver was chosen for a variety of reasons. First of all, it is generally preferable to use different materials for waveguide and plunger in order to minimize the chance of adherence. Because our ambition was to achieve the best possible accuracy with the equipment available, nickel silver was considered because of its distinguished workability on the machines of our mechanical workshop. Finally, the thermal expansion coefficient of nickel silver $\left(16.5 \times 10^{-6} / \mathrm{K}\right)$ is very similar to that of stainless steel $1.4301\left(16 \times 10^{-6} / \mathrm{K}\right)$, which is the target material for the beam-steered antenna. For evaluation of the sliding short's electrical properties, three flat washers with different thicknesses were manufactured from nickel silver. The thickness of each washer has been verified by micrometer gauge measurements with an error of $\pm 5 \mu \mathrm{m}$. The measured thicknesses are given in Tab. I. For S-parameter characterization of the short, a single washer was placed between the reference plane of the sliding short and the flange face. A rubber band realized a certain contact pressure between the flange face, the washer, and the sliding short. This enables a well-defined and accurate positioning of the sliding short relative to the waveguide end. Measurements were carried out on an HP 8510C network analyzer with millimeter-wave extensions which was calibrated with thru-reflect-line (TRL) standards.

In Fig. 9, the reflectivity of the sliding short obtained by simulation and measurement is depicted together with a reference measurement of a static calibration kit short. In the simulation, ideally smooth surfaces were assumed. Hence, the effect of surface roughness is not included. Ripples in the measured data are due to flange inaccuracies (compare with Fig. 14, $\left|s_{21}\right|$ of phase shifter prototype with a correct flange). Because of the larger Ohmic losses in the fabricated prototype due to surface roughness, the simulated reflectivity is the best. The reflectivity of the sliding short varies slightly with frequency but can be considered independent of the plunger position. In the measurement of the calibration kit short, losses are even higher because it was mounted on the flange face and that introduced an additional chunk of (lossy) waveguide. The calibration kit short was mounted at a distance of $50 \mathrm{~mm}$ and the sliding short position $\ell=0 \mathrm{~mm}$ was located at a distance of $40.2 \mathrm{~mm}$ from the TRL-calibrated reference plane. From the measurement of the calibration kit short, a waveguide attenuation per length can be deduced. With this attenuation, the sliding short reflection can be corrected according to its distance from the reference plane. This leads to a mean reflection of the sliding short of $-0.02 \mathrm{~dB}$ at $\ell=0 \mathrm{~mm}$, while the minimal reflection due to the ripples is larger than $-0.10 \mathrm{~dB}$ over the full $W$-band. This is comparable to state-of- 


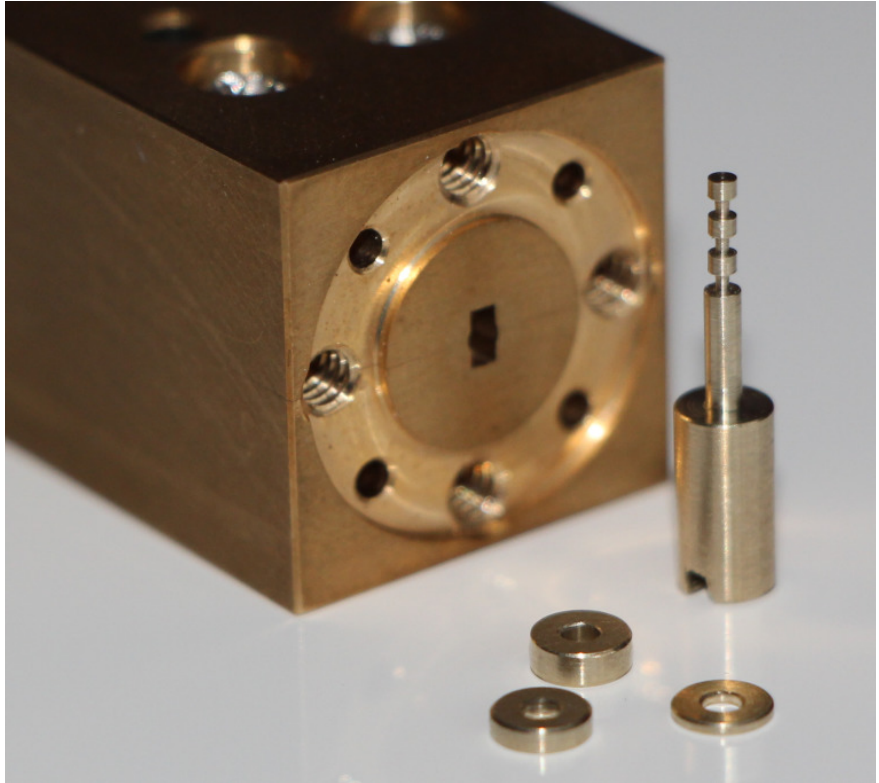

Figure 7. Photograph of the prototyped sliding short. The WR 10 waveguide is manufactured from brass in split-block technology. The guiding fitting hole was drilled and reamed after milling the waveguide. The plunger was manufactured on a lathe from nickel silver. Three flat washers (also nickel silver) with thicknesses $0.5 \mathrm{~mm}, 1.0 \mathrm{~mm}$ and $1.5 \mathrm{~mm}$ were used for precise and reproducible positioning of the plunger during measurements.

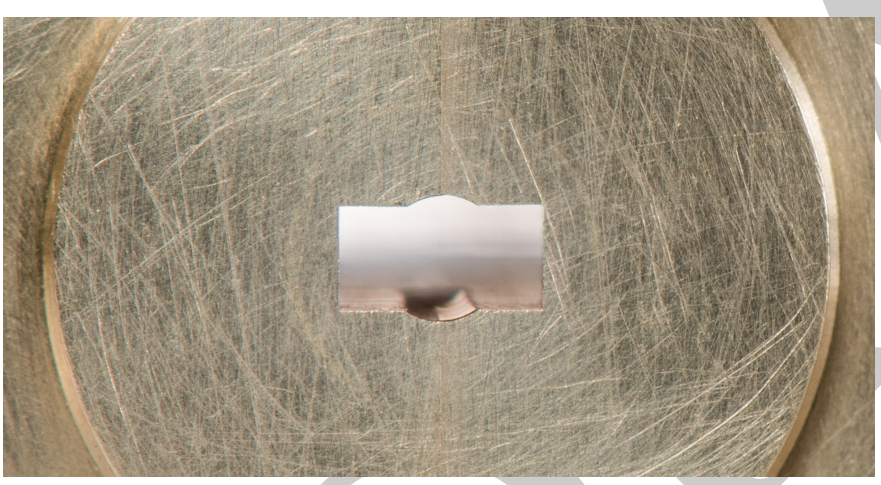

Figure 8. Detailed view of the fitting groove in the rectangular waveguide (bright color is background lighting).

the-art sliding shorts, e.g. [23]. From the simulations (Fig. 3), a value between $-0.02 \mathrm{~dB}$ and $-0.04 \mathrm{~dB}$ is expected. From the measured reflection of the calibration kit short, a conductivity of the realized waveguide with $10.5 \times 10^{6} \mathrm{~S} / \mathrm{m}$ was obtained. The deviation from the information given in the material data sheet $\left(15.9 \times 10^{6} \mathrm{~S} / \mathrm{m}\right)$ is acceptable and can be explained by surface roughness. A further simulation of the prototype with the realized conductivity is also shown in Fig. 9, which agrees very well with the measurement results.

The realized phase difference for the individual reference plane offsets $0.5 \mathrm{~mm}, 1.0 \mathrm{~mm}$ and $1.5 \mathrm{~mm}$ from the flange face are depicted in Fig. 10. The displayed phase represents the difference between the phase at the reference position $\ell$ and the flange face at $\ell=0 \mathrm{~mm}$. Apart from small ripple, which is most likely a pointer to calibration or flange inaccuracies, the measured phase difference agrees very well with what is to be expected from theory with $\Delta \varphi=-2 \beta_{10} \Delta \ell$. Here, $\beta_{10}$ is

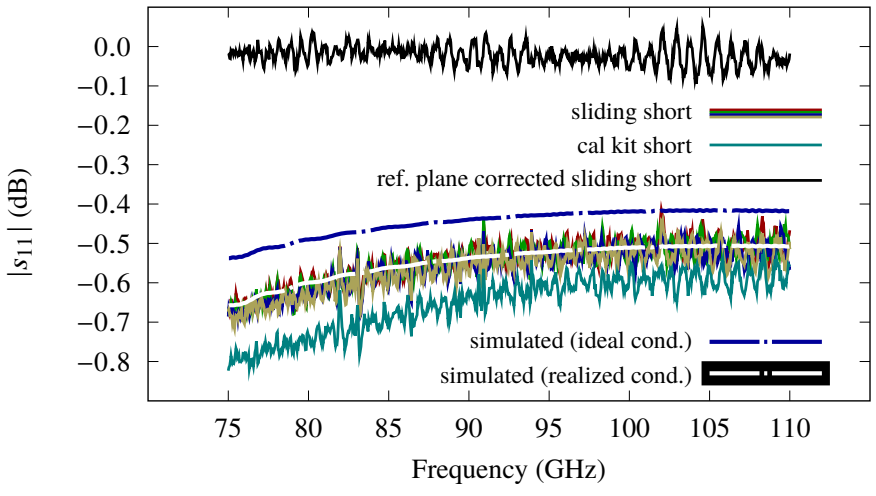

Figure 9. Simulated and measured $\left|s_{11}\right|$ of the periodic plunger sliding short. The simulated short with ideal conductivity $\left(15.9 \times 10^{6} \mathrm{~S} / \mathrm{m}\right)$ has strongest reflection. Measured values are taken at plunger positions $0 \mathrm{~mm}, 0.5 \mathrm{~mm}, 1 \mathrm{~mm}$ and $1.5 \mathrm{~mm}$ - all overlaid. The calibration kit short has the lowest reflection as it is mounted at a larger distance from the reference plane. From the calibration kit short measurement, the realized conductivity $\left(10.5 \times 10^{6} \mathrm{~S} / \mathrm{m}\right)$ was obtained and used for a second simulation. The waveguide attenuation from the measurement reference plane to $0.1 \mathrm{~mm}$ ahead of the sliding short has been removed in the reference plane corrected line. Ripples in the measured data are due to flange inaccuracies.

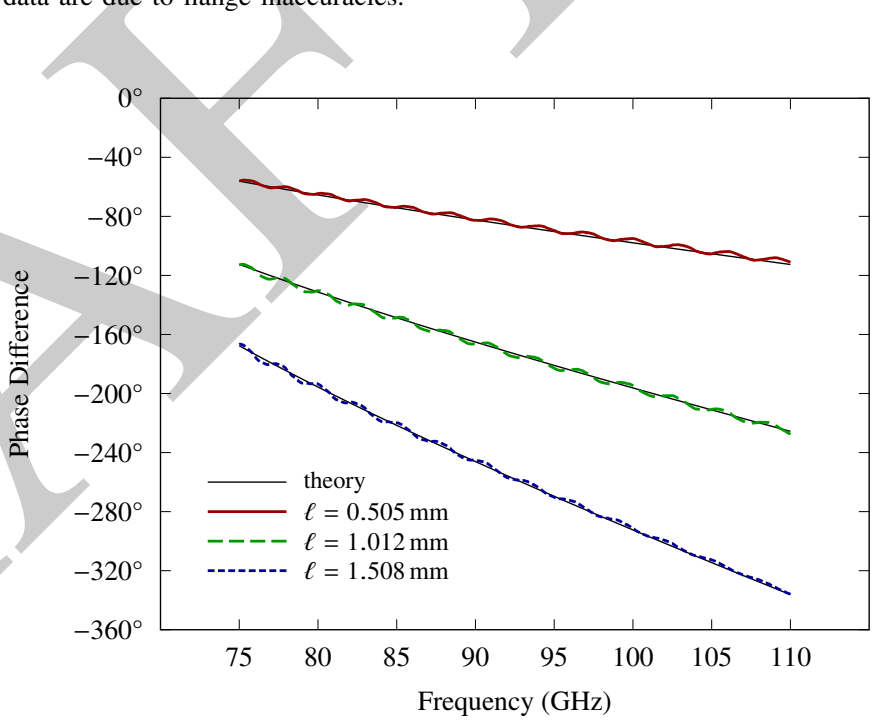

Figure 10. Measured phase difference compared to the theoretically expected behavior between the displayed reference plane positions and the flange face at $\ell=0 \mathrm{~mm}$. Ripple is presumably due to calibration or flange inaccuracies.

the phase constant of a $\mathrm{TE}_{10}$ wave in a WR 10 waveguide and $\Delta \ell$ is the physical offset between the individual short positions that results in the measurable phase difference $\Delta \varphi$. The factor of 2 appears as a result of two-way propagation (forth and back) of the wave.

In Tab. I, the mechanically measured washer thickness and the offset position retrieved from the phase measurement are compared with each other. The measurement of the reference offset position (i.e. the washers) has been carried out with a micrometer gauge $( \pm 5 \mu \mathrm{m}$ error) and the reconstructed offset positions have been found numerically based on the relation $\Delta \ell=\Delta \varphi /\left(2 \beta_{10}\right)$ between physical and electrical length. Measured and reconstructed offset positions agree to a maximum deviation of $6 \mu \mathrm{m}$, which is in the range of the manufacturing tolerance and measurement errors. 
Table I

POSITION OFFSET FROM MECHANICAL MEASUREMENT AND AFTER RETRIEVAL FROM REFLECTED PHASE MEASUREMENT.

\begin{tabular}{cc}
\hline Mechanically measured & Retrieved from reflected phase \\
\hline $0.505 \mathrm{~mm}$ & $0.499 \mathrm{~mm}$ \\
$1.012 \mathrm{~mm}$ & $1.014 \mathrm{~mm}$ \\
$1.508 \mathrm{~mm}$ & $1.505 \mathrm{~mm}$ \\
\hline
\end{tabular}

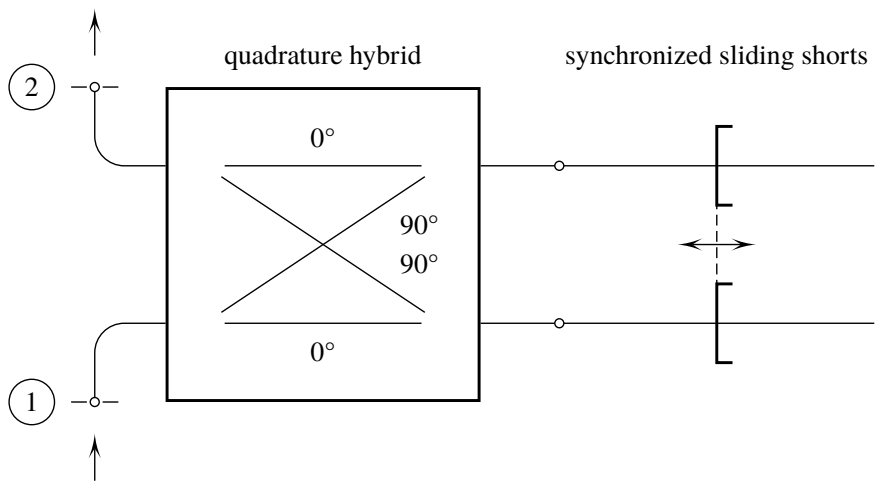

Figure 11. Schematic of the proposed reflection-type phase shifter. Left side represents the quadrature hybrid. On the right side are two collocated and synchronously moved sliding shorts.

\section{Phase Shifter Design}

The considered phase shifter is of reflection-type, where the reflections of two collocated and synchronously moved sliding shorts are transformed into an almost perfect transmission by means of a quadrature hybrid, see Fig. 11. In brief, an incident wave (lower left) is equally split by the quadrature hybrid into two branches. The upper branch has a phase delay of $90^{\circ}$ compared to the lower branch. The waves in both branches get reflected by a sliding short, while both individual sliding shorts are synchronized, i.e. are positioned and moved at the same distance from the quadrature hybrid. The returning waves at the quadrature hybrid interfere constructively at the output (upper left) port as the waves of both channels have experienced the $90^{\circ}$ phase shift and, thus, are in-phase. At the input port (lower left), the wave coming from the upper sliding short has now a $180^{\circ}$ relative phase shift compared to the wave in the lower branch, resulting in destructive interference. In summary, there is a variable phase shift due to the position of the synchronized sliding shorts and a constant $90^{\circ}$ phase shift due to the quadrature hybrid. By varying the position of the sliding shorts, the phase shift can be controlled. The constant $90^{\circ}$ phase shift is important when the electrical length between input and each radiating element of the phased array antenna needs to be equalized.

The quadrature hybrid is realized as a branch-guide coupler. It can achieve more than one octave bandwidth and it can be manufactured in milled split-block technique [19], [27]. A branch-guide coupler consists of two parallel main waveguides and several interconnecting branch-guides. Increasing the number of branches yields better electrical performance [27]. The length and the spacing of the individual branches is a quarter wavelength of the guided wave and their waveguide height determines the coupling coefficient [18], [19], [27]. Due to junction effects, the length of the branches generally needs to be slightly shorter than the ideal quarter wavelength [28]. In literature, two types of branch-guide coupler designs may be distinguished: those that keep the main guide impedances constant and possibly vary the impedances of the individual branches [7], [18] and those that also adjust the main guide impedance according to the branch guide impedance throughout the coupler [19], [27]. While the former type tries to minimize reflection and maximize directivity, the latter type is able to shape/optimize the pass-band performance of the coupler for a certain filter characteristic (e.g. Butterworth or Chebyshev) [19]. The performance of the latter type is superior to that of the former type, but manufacturing is more challenging [27].

For a $W$-band branch-guide coupler in milled split-block technology, the limiting factor for performance is the smallest millable slot. The reason for this is the dependency of the branch-guide coupler performance on the number of branches (more branches, better performance [27]). However, increasing the number of branches comes along with reducing the waveguide height of the individual branches in order to keep the coupling coefficient constant. The smallest possible branch height in our mechanical workshop is $0.200 \mathrm{~mm}$ for a cutting depth of $1.270 \mathrm{~mm}$ ( $W$-band waveguide in splitblock technology). From the tables in [19], a four-branch $3 \mathrm{~dB}$ coupler with either Butterworth or Chebyshev characteristic would have a suitable smallest slot size. However, the height of the inner two slots would be larger than the quarter wave spacing and, hence, would leave no space for the metal inbetween them. In addition, the main guide height of the second section is twice the height of the fundamental waveguide. This introduces further difficulties when placing several phase shifters closely side by side. Based on these considerations, the employed branch-guide coupler is based on a design that keeps the main guide height constant. In particular, it is based on the design presented in [18] with seven slots. The initial branch heights were obtained by the formulas given by Reed [18], which are $192 \mu \mathrm{m}$ for the two outermost branches and $362 \mu \mathrm{m}$ for the five inner ones. By design, the length and the spacing of the individual branches should be $\lambda_{\mathrm{g}} / 4=1.100 \mathrm{~mm}$. Due to the junction effects, the length of the branches is reduced by a factor of $2 d^{\prime}$, while $d^{\prime}$ can be calculated with equation (7) given in $[28, p .338]$. The resulting slot length is $0.760 \mathrm{~mm}$ for the $0.362 \mathrm{~mm}$ slot and $0.840 \mathrm{~mm}$ for a $0.191 \mathrm{~mm}$ slot. The junction effect on the branch spacing is on the order of $0.002 \mathrm{~mm}$. With these initial values, the branch-guide coupler has been optimized using version 2015.6 of CST Microwave Studio ${ }^{\circledR}$. In the simulation model, the two coupled ports are short circuited as they are in the phase shifter application (see Fig. 12) and the $0.191 \mathrm{~mm}$ slots were set to $0.200 \mathrm{~mm}$. The goal of the optimization was not to exceed a reflection of $-20 \mathrm{~dB}$ from $75 \mathrm{GHz}$ to $105 \mathrm{GHz}$. The resulting dimensions are $200 \mu \mathrm{m}$ outer slot height, $340 \mu \mathrm{m}$ inner slot height, $0.81 \mathrm{~mm}$ branch length (applies to all branches), and, $1.07 \mathrm{~mm}$ spacing between the individual branches. 


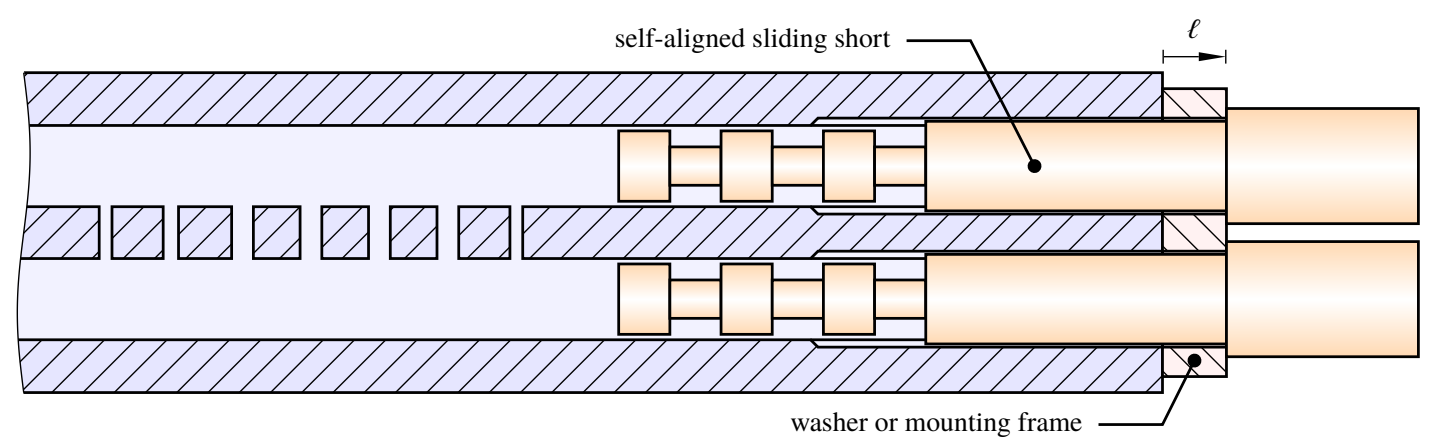

Figure 12. Drawing of the reflection-type phase shifter (cut through the E-plane). On the left side is the seven slot branch-guide coupler. Two self-aligned sliding shorts follow subsequently. A washer or a mounting frame controls the steering position $\ell$. Both sliding shorts are synchronized, i.e. they share the same reference plane. There is no need to externally align the sliding shorts.

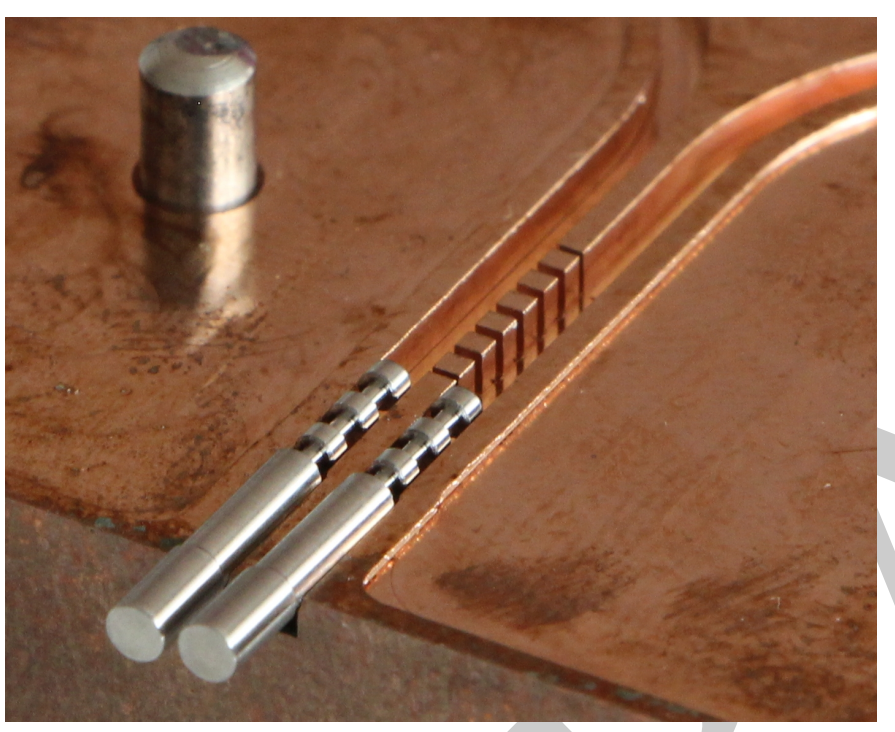

Figure 13. Photograph of the reflection-type phase shifter. The WR 10 waveguide and $3 \mathrm{~dB}$-hybrid are manufactured from Albromet W-200 [29] $\left(22.0 \times 10^{6} \mathrm{~S} / \mathrm{m}\right)$ in split-block technology. The guiding fitting hole was milled with a cherry. The plunger was manufactured on a lathe from stainless steel $1.4301\left(1.37 \times 10^{6} \mathrm{~S} / \mathrm{m}\right)$. Four flat washers with thicknesses $0.5 \mathrm{~mm}, 1.0 \mathrm{~mm}, 1.5 \mathrm{~mm}$ and $2.0 \mathrm{~mm}$ were used for precise and reproducible positioning of the plunger during the measurements.

\section{PhASE ShifTer Prototype}

The branch-guide coupler has been manufactured in milled split-block technology from Albromet W-200 [29]. This material is compatible with the in-vessel environmental conditions in the ASDEX Upgrade tokamak in Garching, Germany. A photograph of the phase shifter prototype is shown in Fig. 13. In the lower left of Fig. 13 are the two sliding shorts as described in section II placed in their individual fitting holes. For this prototype, they are manufactured from stainless steel 1.4301. The other two ports of the branch-guide coupler in Fig. 13 are input and output port.

The measured transmission and reflection magnitudes of the phase shifter are shown in Fig. 14. The measurements were carried out on a HP $8510 \mathrm{C}$ network analyzer with $W$ band extensions calibrated with thru-reflection-line (TRL) standards. The measurement of a calibration kit standard waveguide section showed a reflection below $-40 \mathrm{~dB}$. Four different sliding short positions were realized with washers $(0.526 \mathrm{~mm}, 0.993 \mathrm{~mm}, 1.508 \mathrm{~mm}$, and $2.034 \mathrm{~mm})$ as these provide an accurate and repeatable positioning. The $0.526 \mathrm{~mm}$ washer was used as reference for the three different relative phase shifts of the other washers. The upper plot in Fig. 14 shows the $\left|s_{21}\right|$ which includes Ohmic losses of the $68 \mathrm{~mm}$ waveguide from the input measurement flange to the branchguide coupler and from the branch-guide coupler to the output measurement flange. These losses are removed from the raw data in the central plot of Fig. 14. The realized conductivity has been obtained from a $50 \mathrm{~mm}$ reference section machined in the same split-block together with the phase shifter (ideal conductivity of Albromet W-200: $22 \times 10^{6} \mathrm{~S} / \mathrm{m}$, realized conductivity: $12.3 \times 10^{6} \mathrm{~S} / \mathrm{m}$ ). The lower plot in Fig. 14 shows the simulated and measured $\left|s_{11}\right|$. Comparing the $\left|s_{21}\right|$ with the full-wave simulation (version 2016.4 of CST Microwave Studio ${ }^{\circledR}$, reference plane $0.1 \mathrm{~mm}$ apart from phase shifter for the simulation in the central plot of Fig. 14) shows perfect agreement with the measured values when using the realized conductivity. The phase shifter operates as desired in the frequency range from $80 \mathrm{GHz}$ to $105 \mathrm{GHz}$ with an insertion loss in the range from $0.2 \mathrm{~dB}$ to $0.4 \mathrm{~dB}$ for all sliding short positions. From $75 \mathrm{GHz}$ to $80 \mathrm{GHz}$ the insertion loss increases. A study of the phase shifter by full-wave simulations underpins the assumption that the height of the coupling slots has been realized slightly larger than the specified heights, leading to the observed behavior of the S-parameters.

The realized phase shifts of the $0.993 \mathrm{~mm}, 1.508 \mathrm{~mm}$, and $2.034 \mathrm{~mm}$ offset positions are shown in Fig. 15 with reference to the $0.526 \mathrm{~mm}$ offset. The phase error compared to the theoretical value is $20^{\circ}$ at maximum for the $1.5-\mathrm{mm}$ relative offset at $75 \mathrm{GHz}$. All other deviations are in the range from $+8^{\circ}$ to $-6^{\circ}$. In Tab. II, the mechanically measured offset position and that retrieved from the reflected phase are listed. For this measurement, the uncertainty of the mechanically measured offset position is $\pm 10 \mu \mathrm{m}$ as the reference was not the fixed flange face but another washer. The maximum difference between the mechanically measured and the offset retrieved from the reflected phases is $10 \mu \mathrm{m}$, which is in the range of manufacturing tolerance and measurement errors. A continuous phase shift is possible by using a Piezo actuator instead of the static washer for the positioning of the sliding 

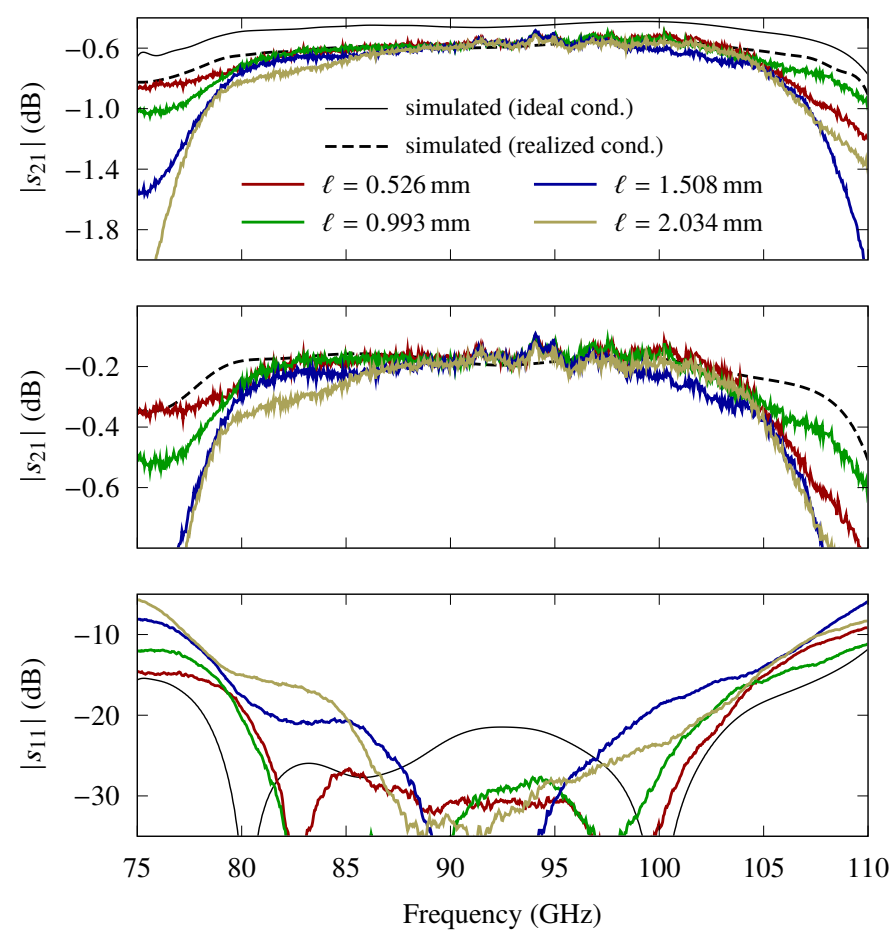

Figure 14. The upper plot shows simulated and measured $\left|s_{21}\right|$ results with approx. $68 \mathrm{~mm}$ lossy waveguide from the measurement reference planes to the phase shifter. Simulated data is plotted for the ideal conductivity $\left(22 \times 10^{6} \mathrm{~S} / \mathrm{m}\right)$ and the realized conductivity $\left(12.3 \times 10^{6} \mathrm{~S} / \mathrm{m}\right)$, which was obtained from a $50 \mathrm{~mm}$ reference waveguide. The central plot shows $\left|s_{21}\right|$ with a reference plane $0.1 \mathrm{~mm}$ away from the phase shifter. The measured data was obtained by removing the $68 \mathrm{~mm}$ waveguide attenuation from the raw data. The lower plot shows the $\left|s_{11}\right|$.

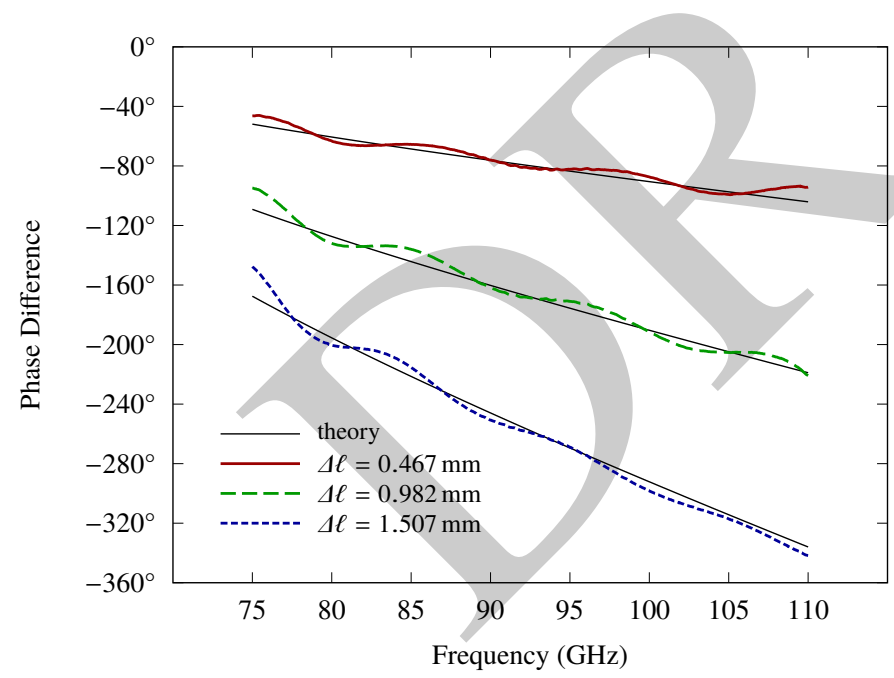

Figure 15. Measured phase difference of the $1 \mathrm{~mm}, 1.5 \mathrm{~mm}$ and $2 \mathrm{~mm}$ sliding short position with reference to the $0.5 \mathrm{~mm}$ position. The theoretically expected behavior is shown for comparison.

shorts.

\section{CONCLUSION}

A self-aligning all-metal sliding short design was presented. The incident wave is reflected from a coaxial choke structure, which carries an evanescent wave. The sliding plunger is composed of three alternating low-impedance high-impedance
Table II

POSITION OFFSET FROM MECHANICAL MEASUREMENT AND AFTER RETRIEVAL FROM PHASE MEASUREMENT.

\begin{tabular}{cc}
\hline Mechanically measured & Retrieved from phase measurement \\
\hline $0.467 \mathrm{~mm}$ & $0.457 \mathrm{~mm}$ \\
$0.982 \mathrm{~mm}$ & $0.972 \mathrm{~mm}$ \\
$1.507 \mathrm{~mm}$ & $1.516 \mathrm{~mm}$ \\
\hline
\end{tabular}

sections and it is directed in a fitting hole by a subsequent guiding section to prevent it from random and unpredictable contact with the waveguide walls. A mechanical stop is used for a controlled positioning of the sliding short in the waveguide. It is possible to use the proposed sliding short design in phase shifter circuits for phased array antennas, as each plunger is self-aligning with respect to the waveguide cross section and all plungers can share the same reference plane. An empirical full-wave simulation study as well as a $W$-band prototype have proven the expected behavior. The maximum return loss is $0.1 \mathrm{~dB}$ and the plunger position can be set in micrometer accuracy. Based on the proposed sliding short, a reflection-type phase shifter has been designed and prototyped. A branch-guide coupler is used as hybrid junction. Two self-aligning sliding shorts are placed side-by-side in their individual fitting hole. Their position is controlled by means of washers and the mechanical stops of the sliding shorts. The insertion loss is around $0.2 \mathrm{~dB}$ and the positions of the sliding shorts agree well with their expected values.

\section{REFERENCES}

11] R. V. Garver, "Broad-band diode phase shifters," IEEE Trans. Microw. Theory Techn., vol. 20, no. 5, pp. 314-323, May 1972.

[2] G. M. Rebeiz, G.-L. Tan, and J. S. Hayden, "RF MEMS phase shifters: design and applications," IEEE Microw. Mag., vol. 3, no. 2, pp. 72-81, Jun. 2002.

[3] S. Gong, H. Shen, and N. S. Barker, "A 60-GHz 2-bit switched-line phase shifter using SP4T RF-MEMS switches," IEEE Trans. Microw. Theory Techn., vol. 59, no. 4, pp. 894-900, Apr. 2011.

[4] S. Barker and G. M. Rebeiz, "Distributed MEMS true-time delay phase shifters and wide-band switches," IEEE Trans. Microw. Theory Techn., vol. 46, no. 11, pp. 1881-1890, Nov. 1998.

[5] A.-L. Franc, O. H. Karabey, G. Rehder, E. Pistono, R. Jakoby, and P. Ferrari, "Compact and broadband millimeter-wave electrically tunable phase shifter combining slow-wave effect with liquid crystal technology," IEEE Trans. Microw. Theory Techn., vol. 61, no. 11, pp. 3905-3915, Nov. 2013.

[6] D. Parker and D. C. Zimmermann, "Phased arrays-part II: implementations, applications, and future trends," IEEE Trans. Microw. Theory Techn., vol. 50, no. 3, pp. 688-698, Mar. 2002.

[7] P. D. Lomer and J. W. Crompton, "A new form of hybrid junction for microwave frequencies," Proceedings of the IEE - Part B: Radio and Electronic Engineering, vol. 104, no. 15, pp. 261-264, May 1957.

[8] C.-S. Lin, S.-F. Chang, C.-C. Chang, and Y.-H. Shu, "Design of a reflection-type phase shifter with wide relative phase shift and constant insertion loss," IEEE Trans. Microw. Theory Techn., vol. 55, no. 9, pp. 1862-1868, Sep. 2007.

[9] H.-T. Kim, J.-H. Park, J. Yim, Y.-K. Kim, and Y. Kwon, "A compact Vband 2-bit reflection-type MEMS phase shifter," IEEE Microw. Wireless Compon. Lett., vol. 12, no. 9, pp. 324-326, Sep. 2002.

[10] P. Rohmann, S. Wolf, W. Kasparek, B. Plaum, and J. Hesselbarth, "A 32element frequency-steered array antenna for reflectometry in W-band," in IEEE Int. Symp. Phased Array Systems and Technology, Waltham, MA, USA, Oct. 2013, pp. 559-563. 
[11] C. Koenen, U. Siart, T. F. Eibert, G. D. Conway, and U. Stroth, "Broadband amplitude tapering for a linear W-band array antenna for Gaussian beam-shaping," in German Microwave Conf., Nuremberg, Germany, Mar. 2015, pp. 202-204.

[12] — , "Design of a millimeter-wave phased array antenna for Gaussian beam shaping and steering," in IEEE Int. Symp. Phased Array Systems and Technology, Waltham, MA, USA, Oct. 2016.

[13] G. D. Conway, E. Poli, T. Happel, and the ASDEX Upgrade Team, "Interaction of mean and oscillating plasma flows across confinement mode transitions," Plasma and Fusion Research, vol. 5, p. S2005, 2010.

[14] U. Stroth et al., "Experimental turbulence studies for gyro-kinetic code validation using advanced microwave diagnostics," Nuclear Fusion, vol. 55, no. 8, p. 083027, Aug. 2015.

[15] M. Hirsch, E. Holzhauer, J. Baldzuhn, B. Kurzan, and B. Scott, "Doppler reflectometry for the investigation of propagating density perturbations," Plasma Physics Controlled Fusion, vol. 43, no. 12, pp. 1641-1660, 2001.

[16] G. D. Conway, "Turbulence measurements in fusion plasmas," Plasma Physics Controlled Fusion, vol. 50, no. 12, p. 124026, 2008.

[17] T. Happel et al., "Design of a new Doppler reflectometer frontend for the ASDEX Upgrade tokamak," in 10th Int. Reflectometry Workshop, Padova, Italy, May 2011

[18] J. Reed, "The multiple branch waveguide coupler," IRE Trans. Microw. Theory Techn., vol. 6, no. 4, pp. 398-403, Apr. 1958.

[19] R. Levy, "Zolotarev branch-line couplers," IEEE Trans. Microw. Theory Techn., vol. 21, no. 2, pp. 95-99, Feb. 1973

[20] M. K. Brewer and A. V. Räisänen, "Dual-harmonic noncontacting millimeter waveguide backshorts: Theory, design, and test," IEEE Trans. Microw. Theory Techn., vol. 30, no. 5, pp. 708-714, May 1982.

[21] A. R. Kerr, "An adjustable short-circuit for millimeter waveguides," National Radio Astronomy Observatory, USA, Electronics Division Internal Report 280, Jul. 1988.

[22] T. Newman and K. T. Ng, "Planar noncontacting short circuits for millimeter-wave and submillimeter-wave applications," IEEE Microw. Guided Wave Lett., vol. 2, no. 10, pp. 412-414, Oct. 1992.

[23] J. R. Stanec and N. S. Barker, "A rectangular-waveguide contacting sliding short for terahertz frequency applications," IEEE Trans. Microw. Theory Techn., vol. 61, no. 4, pp. 1488-1495, Apr. 2013.

[24] W. R. McGrath, T. M. Weller, and L. P. B. Katehi, "A novel noncontacting waveguide backshort for submillimeter wave frequencies," Int. J. Infrared Millimeter Waves, vol. 16, no. 1, pp. 237-256, 1995.

[25] T. M. Weller, L. P. B. Katehi, and W. R. McGrath, "Analysis and design of a novel noncontacting waveguide backshort," IEEE Trans. Microw. Theory Techn., vol. 43, no. 5, pp. 1023-1030, May 1995.

[26] V. S. Möttönen, P. Piironen, and A. V. Räisänen, "Novel tunable waveguide backshort for millimeter and submillimeter wavelengths," IEEE Microw. Wireless Compon. Lett., vol. 11, no. 9, pp. 370-372, Sep. 2001.

[27] L. Young, "Synchronous branch guide directional couplers for low and high power applications," IRE Transactions on Microwave Theory and Techniques, vol. 10, no. 6, pp. 459-475, Nov. 1962.

[28] N. Marcuvitz, Waveguide Handbook, ser. Radiation Laboratory Series. New York: McGraw-Hill Book Company, Inc., 1951, vol. 10.

[29] Albromet GmbH, Geretsried, Germany, http://www.albromet.org/, visited on $08 / 01 / 2016$.

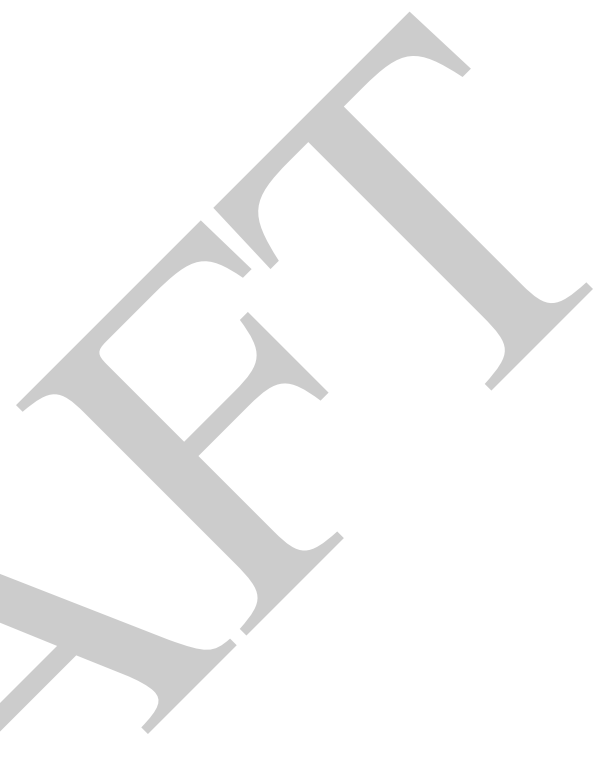

